\title{
Design and Enhancement of Distributed Architecture in Health Insurance System
}

\author{
R.Sriram 1 , Dr J. Andrews² \\ 1,2Sathyabama University, Chennai, TamilNadu
}

\begin{abstract}
Background: The main objective of this paper is to review the literature about Distributed Architecture in Health Insurance System. Health Axis System involves major factors for applying Asp.Net Web Applications, Different Authentication, Authorization and Framework Techniques, covered for Different Coding Techniques. Methods and Statistical Analysis: This Paper is explained about distributed architecture online health Insurance. Health Axis Information about all sort of medical services providing by the hospital to corporate companies, claim settlements \& adjustments services authorized by insurance companies. Findings: Experiment is analyzed to Create Secured Frame Implement Secured Health Insurance System. The Experimental Program Code Result is given to proposed method. Conclusion: In This paper give the point by point review of different methods and Techniques with coding structures to create Secured Architecture inAsp.Net Web Applications.
\end{abstract}

Keywords: Primary health Care, Claim Processing, adjudication, Quality healthcare, Co-Insurance, Premium

\section{INTRODUCTION}

Most of the Health Insurance System developed by different vendors and develop different plat forms. Interoperability is the ability of different subsystems to access and use the data reliably and quickly from various sources without the occurances ${ }^{1}$. Estimating the overall risk of health care expenses, among a targeted group, an insurer can develop a routine finance structure, such as a monthly premium or payroll tax; ensure that money is available to pay for the health care benefits specified in the insurance agreement1. The benefit is administered by a central organization such as a government agency, private business, or not-for-profit entity. In the current healthcare scenario, patients have multiple health records in different healthcare information systems. Therefore interoperability in healthcare information systems ever more becomes a requirement rather than a feature.

\section{TECHNOLOGY OVERVIEW}

The most suitable approach to handle the interoperability issue among the crucial medicinal services segments in appropriated human services environment is blend of smart specialists and case based thinking. The unmistakable elements of each, gainful for the proposed framework, are portrayed underneath'.

\section{A. Client Layer}

It is the Presentation Layer where in which the user interface for the client built.

\section{B. Application Logic Layer}

Application logic insurance engine is built using Asp.Net, MVC, and COM Components. A request may be fetching information, storing information, searching information?2.

The Client request to the Server Engine the WEB API Processing the request to Database Layer and back to UI Layer 1,2 .

\section{Data Layer}

The Engine uses SQL Server 2012 or Cloud SQL Database for Persistence storage information. Insurance database representing data layer.

\section{PROBLEMS IN THE EXISTING SYSTEM}

1. Limited in Single System. (Need to execute Service Oriented Architecture) $)^{3}$.

2. Fewer clients Friendly.

3. Having part of manual work (Manual System does not imply that you are working with pen and paper, it additionally incorporate taking a shot at spread sheets and other basic programming). 
4. Requires more number of representative's to work. (Not computerized)

5. Its time consuming process.

6. Less Storage for existing System.

7. Less performance.

8. Claim Processing will less Secure like HIFA and FIFA Processing ${ }^{3}$.

9. Every day Batch Process not implemented.

10. Track record its very tedious process.

11. Less secured for existing system in Database(SQL Injection Attack.

\section{GOAL OF THE PROPOSED SYSTEM}

This task is extremely famous and generally utilized as a part of numerous enormous associations. This venture is meant to building up an online medical coverage against its worker. This is an online framework and is utilized keep up the vast majority of the exercises happening in enormous associations ${ }^{4}$. The Health Axis framework will keep up all data identified with the representative, support of cases relating (Batch procedure) to the insurance agencies, data about all kind of medicinal administrations giving by the doctor's facility to the corporate workers, claim settlements and changes against their administrations writing by the protection companies $^{5}$. Furthermore this framework can likewise keep up the wellbeing related all data of the employees ${ }^{5}$

\section{SOLUTION OF THESE PROBLEMS}

The improvement of the new framework contains the accompanying exercises, which attempt to computerize the whole process keeping in perspective of the database coordin 5 .

1. The application with various controls (Telerik, Ajax, Anjular JS for validations, version control Tortoise workbench).

2. Host the application Cloud Computing access through VMWare

3. The system makes the overall project management much easier and flexible.

4. Various custom components used to implement entire application's Like (AdRotator, JSGrid, WorkBenchTools, CustomCalender control Creation, Autoevent Environment ect..).
5. There is no risk data management at any level while the project development is under process.

6. It Provides high level Protocols using secured manner.

7. Implement highly secured architecture for MVC Security controls.

8. To implements WebApi once complete the project to implement different client locations to Service only.

9. Increase DataBase storage space to implement SQL Server or BIGData, Cloud SQL.

10. The System To implement Cloud Environment to access everywhere for performance will increase.

11. To Implement (BATCH Process for the Entire System). it's used for automatically track the track efficient manner.

\section{STUDY OF THE SYSTEM}

In the adaptability of the utilizations the interface has been created a design idea at the top of the priority list, related through a program interface. The GUI'S at the top level have been classified $a^{5}$.

1. Administrative User Interface.

2. Employee User Interface.

3. Claim Generation User Interface.

4. Batch Process User Interface.

5. The regulatory client interface focuses on the reliable data that is for all intents and purposes, part of the hierarchical exercises and which needs appropriate confirmation for the information gathering 4,5 . The interfaces help the organizations with all the value-based states like Data insertion, Data erasure and Data redesigning alongside the broad information seek capabilities ${ }^{5}$.

6. The operational or non specific client interface helps the clients upon the framework in exchanges through the current information and required services ${ }^{6}$. The operational client interface likewise offers the normal clients in dealing with their own data some assistance with helping the conventional clients in dealing with their own data in an altered way according to the helped adaptabilities 6,7 . 


\section{ARCHITECTURAL AND DESIGN FEATURES}

\begin{tabular}{|c|c|c|}
\hline $\begin{array}{l}\text { Sr. } \\
\text { No }\end{array}$ & $\begin{array}{c}\text { Architecture/Design } \\
\text { Feature }\end{array}$ & Description \\
\hline 1 & \multicolumn{2}{|c|}{ Presentation } \\
\hline 1.1 & HTML/ASPX Pages & $\begin{array}{l}\text { Use .cshtml with Razor } \\
\text { Syntax }\end{array}$ \\
\hline 1.2 & $\begin{array}{l}\text { Cascade style } \\
\text { sheets (CSS), } \\
\text { Themes, Skins }\end{array}$ & Style Definations \\
\hline 1.3 & Images and Logos & $\begin{array}{l}\text { File system/get from the } \\
\text { website folders; }\end{array}$ \\
\hline 1.4 & Clientside validations & $\begin{array}{l}\text { jQuery, MVC and Data } \\
\text { Annotations }\end{array}$ \\
\hline 1.5 & $\begin{array}{l}\text { jQuery } \\
\text { controls/Plugins }\end{array}$ & Identify jQuery Plugins \\
\hline 1.6 & $\begin{array}{l}\text { Third party custom } \\
\text { controls }\end{array}$ & $\begin{array}{l}\text { Telerik MVC/jQuery } \\
\text { based grid controls }\end{array}$ \\
\hline 1.7 & Navigation & $\begin{array}{l}\text { Use CSS based Top } \\
\text { Menu, Left side } \\
\text { Navigation }\end{array}$ \\
\hline 1.8 & Alerts and Messages & $\begin{array}{l}\text { Use jQuery Modal for } \\
\text { alerts. jQuery Modal } \\
\text { gives flexibility over the } \\
\text { interface and actions }\end{array}$ \\
\hline 1.9 & Popup windows & $\begin{array}{l}\text { Use jQuery Modal to } \\
\text { provide popup } \\
\text { functionality; Need } \\
\text { customization options } \\
\text { like minimize, drag, } \\
\text { maximize where required }\end{array}$ \\
\hline 1.10 & AJAX & $\begin{array}{l}\text { Validation, load data on } \\
\text { demand }\end{array}$ \\
\hline 1.11 & jQuery Server calls & $\begin{array}{l}\text { JSON/RESTful services, } \\
\text { Use jQuery .ajax function } \\
\text { with }\end{array}$ \\
\hline 1.12 & $\begin{array}{l}\text { Page Refresh } \\
\text { Full/Partial }\end{array}$ & $\begin{array}{l}\text { Use AJAX for Partial } \\
\text { Refresh }\end{array}$ \\
\hline 1.13 & Browser compatibility & $\begin{array}{l}\text { Target IE 7/8/9, Firefox, } \\
\text { Chrome, Safari, } \\
\text { Modernizr. Use CSS } \\
\text { Reset to avoid cross- } \\
\text { browser differences }\end{array}$ \\
\hline 1.14 & $\begin{array}{l}\text { User Experience and } \\
\text { Usability }\end{array}$ & User Friendly \\
\hline 1.15 & Data Pagination & Load on scroll \\
\hline 1.16 & Mobile UI & Mobility UI \\
\hline
\end{tabular}

\begin{tabular}{|c|c|c|}
\hline $\begin{array}{l}\text { Sr. } \\
\text { No }\end{array}$ & $\begin{array}{c}\text { Architecture/Design } \\
\text { Feature }\end{array}$ & Description \\
\hline 1.17 & Tablet UI & Tablet UI Design \\
\hline 1.18 & Sitemap & Global Site Map \\
\hline 2 & \multicolumn{2}{|c|}{ Business Logic } \\
\hline 2.1 & $\begin{array}{l}\text { Business Rules and } \\
\text { Validations }\end{array}$ & $\begin{array}{l}\text { Configure Business } \\
\text { Rules by Customer and } \\
\text { stored in Meta Data } \\
\text { Services database }\end{array}$ \\
\hline 2.2 & $\begin{array}{l}\text { Business } \\
\text { Implementation } \\
\text { Classes }\end{array}$ & Business Layer \\
\hline 2.3 & $\begin{array}{l}\text { Data Transfer } \\
\text { Objects }\end{array}$ & $\begin{array}{l}\text { Make them as close as } \\
\text { possible to Database, } \\
\text { Interface on DTOs, Data } \\
\text { Mappers }\end{array}$ \\
\hline 2.4 & WCF Services & $\begin{array}{l}\text { Acts as interface } \\
\text { between different } \\
\text { environment }\end{array}$ \\
\hline 2.5 & $\begin{array}{l}\text { Interfaces } \\
\text { (Components) }\end{array}$ & $\begin{array}{l}\text { Communicate for each } \\
\text { Layered Structure }\end{array}$ \\
\hline 2.6 & Data annotations & $\begin{array}{l}\text { DataRegion Specific } \\
\text { View Models/Separate } \\
\text { Views By State }\end{array}$ \\
\hline 2.7 & Business Workflows & $\begin{array}{l}\text { Identify Business } \\
\text { Workflows }\end{array}$ \\
\hline 2.8 & Service Contracts & $\begin{array}{l}\text { Define service operations } \\
\text { that service } \\
\text { support/implement }\end{array}$ \\
\hline 2.9 & Data Contracts & $\begin{array}{l}\text { Data contract for } \\
\text { communication between } \\
\text { services and clients }\end{array}$ \\
\hline 3 & $\mathrm{Da}$ & \\
\hline 3.1 & Data access classes & DataBase Layer \\
\hline 3.2 & Stored Procedures & For Performance wise \\
\hline 3.3 & $\begin{array}{l}\text { User defined } \\
\text { functions }\end{array}$ & UI Functions \\
\hline 3.4 & Views & $\begin{array}{l}\text { Multiple user access a } \\
\text { Table }\end{array}$ \\
\hline 3.5 & Triggers & Data Manupulation \\
\hline 3.6 & Database design & $\begin{array}{l}\text { Follow Normalization } \\
\text { Process, etc }\end{array}$ \\
\hline 3.7 & Exception Handling & Error Handling \\
\hline 3.8 & $\begin{array}{l}\text { Concurrency } \\
\text { handling and } \\
\text { Transaction } \\
\text { Management }\end{array}$ & Transaction Management \\
\hline
\end{tabular}




\begin{tabular}{|c|c|c|}
\hline $\begin{array}{l}\text { Sr. } \\
\text { No }\end{array}$ & $\begin{array}{l}\text { Architecture/Design } \\
\text { Feature }\end{array}$ & Description \\
\hline 3.9 & Connection Pooling & $\begin{array}{l}\text { Multiple Instance } \\
\text { Checking }\end{array}$ \\
\hline 3.10 & Data Migration & $\begin{array}{l}\text { Migrate master tables } \\
\text { data }\end{array}$ \\
\hline 3.11 & ADO.NET & DataBase Activities \\
\hline 4 & \multicolumn{2}{|c|}{ Core Framework } \\
\hline 4.1 & $\begin{array}{l}\text { Session } \\
\text { Management }\end{array}$ & $\begin{array}{l}\text { Use Out of Process state } \\
\text { server for session } \\
\text { management }\end{array}$ \\
\hline 4.2 & Exception Handling & $\begin{array}{l}\text { Enterprise Library for } \\
\text { exception handling is } \\
\text { used } \\
\text { Policy will determine } \\
\text { when to log, propagate, } \\
\text { change the original } \\
\text { exception message }\end{array}$ \\
\hline 4.3 & Logging & $\begin{array}{l}\text { Enterprise Library } \\
\text { Logging application block } \\
\text { is used for storing the } \\
\text { exception stack trace. }\end{array}$ \\
\hline 4.4 & Caching & $\begin{array}{l}\text { Use Enterprise Library } \\
\text { for Caching; SQL Cache } \\
\text { Dependency. Use } \\
\text { caching to store the data } \\
\text { for dropdown fields, static } \\
\text { pages } \\
\text { Cache Invalidation }\end{array}$ \\
\hline 4.5 & Authentication & $\begin{array}{l}\text { Forms Authentication will } \\
\text { be used and need to } \\
\text { develop membership } \\
\text { service }\end{array}$ \\
\hline 4.6 & Authorization & $\begin{array}{l}\text { Role based; Dynamic } \\
\text { roles required for testing }\end{array}$ \\
\hline 4.7 & Auto logout & $\begin{array}{l}\text { After a period of } \\
\text { inactivity, user will need } \\
\text { to be logged out } \\
\text { automatically with a } \\
\text { prompt before } 5 \text { minutes } \\
\text { of auto logout }\end{array}$ \\
\hline 4.8 & $\begin{array}{l}\text { Namespace and } \\
\text { Directory structure }\end{array}$ & $\begin{array}{l}\text { Add Difference Name } \\
\text { Space and Components }\end{array}$ \\
\hline 4.9 & Encryption & $\begin{array}{l}\text { Query string parameters } \\
\text { need to be encrypted }\end{array}$ \\
\hline 4.10 & URL Redirection & $\begin{array}{l}\text { Use MVC Redirect } \\
\text { Options }\end{array}$ \\
\hline 4.11 & Handling Query & Always encrypt query \\
\hline
\end{tabular}

\begin{tabular}{|c|c|c|}
\hline $\begin{array}{l}\text { Sr. } \\
\text { No }\end{array}$ & $\begin{array}{c}\text { Architecture/Design } \\
\text { Feature }\end{array}$ & Description \\
\hline & string parameters & string parameter values \\
\hline 4.12 & Auditing & $\begin{array}{l}\text { Detailed information is } \\
\text { logged }\end{array}$ \\
\hline 4.13 & Load Balancing & $\begin{array}{l}\text { Performance Testing } \\
\text { and Review }\end{array}$ \\
\hline 4.14 & Security & $\begin{array}{l}\text { SSL, Authentication, } \\
\text { Resources like Scripts } \\
\text { and Images }\end{array}$ \\
\hline 5 & \multicolumn{2}{|c|}{ Framework/Patterns } \\
\hline 5.1 & MVC & $\begin{array}{l}\text { Presentation design } \\
\text { pattern, extensible to } \\
\text { other devices }\end{array}$ \\
\hline 5.2 & SOA & $\begin{array}{l}\text { Business logic exposed } \\
\text { as services }\end{array}$ \\
\hline 5.3 & $\begin{array}{l}\text { Design Patterns } \\
\text { (Factory, Adapter } \\
\text { e.t.c) }\end{array}$ & $\begin{array}{l}\text { Waterfall, Agile } \\
\text { Methodology }\end{array}$ \\
\hline 5.4 & $\begin{array}{l}\text { Dependency } \\
\text { Injection/loC }\end{array}$ & SQL Dependency \\
\hline 6 & \multicolumn{2}{|c|}{ Deployment Strategy } \\
\hline 6.1 & $\begin{array}{l}\text { Deployment } \\
\text { Environments ( } \\
\text { Alpha, Beta, Staging, } \\
\text { Prod) }\end{array}$ & Build and Release \\
\hline 6.2 & $\begin{array}{l}\text { Virtual Directories / } \\
\text { Sub sites }\end{array}$ & IIS \\
\hline 6.3 & $\begin{array}{l}\text { Deployment } \\
\text { Architecture Cloud }\end{array}$ & Deployment \\
\hline 7 & \multicolumn{2}{|c|}{ Other } \\
\hline 7.1 & $\begin{array}{l}\text { Performance } \\
\text { Requirements }\end{array}$ & $\begin{array}{l}\text { DataBase, Coding } \\
\text { Standard }\end{array}$ \\
\hline 7.2 & Testing automation & Nunit,Manual \\
\hline 7.3 & $\begin{array}{l}\text { Reusable } \\
\text { Components }\end{array}$ & $\begin{array}{l}\text { Telerik, AdRotator, } \\
\text { Infragistics }\end{array}$ \\
\hline 7.4 & \begin{tabular}{|l} 
External System \\
Dependencies \\
\end{tabular} & \\
\hline 7.5 & $\begin{array}{l}\text { Microsoft Enterprise } \\
\text { Library }\end{array}$ & Ado.Net Framework \\
\hline 7.6 & $\begin{array}{l}\text { Coding Standards } \\
\text { and Best Practices }\end{array}$ & $\begin{array}{l}\text { Uniqueness for Coding } \\
\text { Standards }\end{array}$ \\
\hline 7.7 & Code Review Tools & $\begin{array}{l}\text { Nunit for Methods } \\
\text { Checking }\end{array}$ \\
\hline 7.8 & $\begin{array}{l}\text { Detection of Unused } \\
\text { Translations } \\
\end{array}$ & $\begin{array}{l}\text { Translations for new } \\
\text { detection }\end{array}$ \\
\hline
\end{tabular}




\begin{tabular}{|c|c|c|}
\hline $\begin{array}{c}\text { Sr. } \\
\text { No }\end{array}$ & $\begin{array}{c}\text { Architecture/Design } \\
\text { Feature }\end{array}$ & Description \\
\hline 8 & \multicolumn{2}{|c|}{ Configuration Management } \\
\hline 8.1 & Branching and Merging & SVN \\
\hline
\end{tabular}

\begin{tabular}{|c|c|c|}
\hline $\begin{array}{c}\text { Sr. } \\
\text { No }\end{array}$ & $\begin{array}{c}\text { Architecture/Design } \\
\text { Feature }\end{array}$ & Description \\
\hline 8.2 & $\begin{array}{c}\text { Work Items and } \\
\text { Progress Tracking }\end{array}$ & Tracing ,UTrack Tool \\
\hline 8.3 & Automated Builds & Tortoise WorkBench \\
\hline
\end{tabular}

\section{A. DataFlow Diagramatical Claim Process Representation} registeration ID and Password

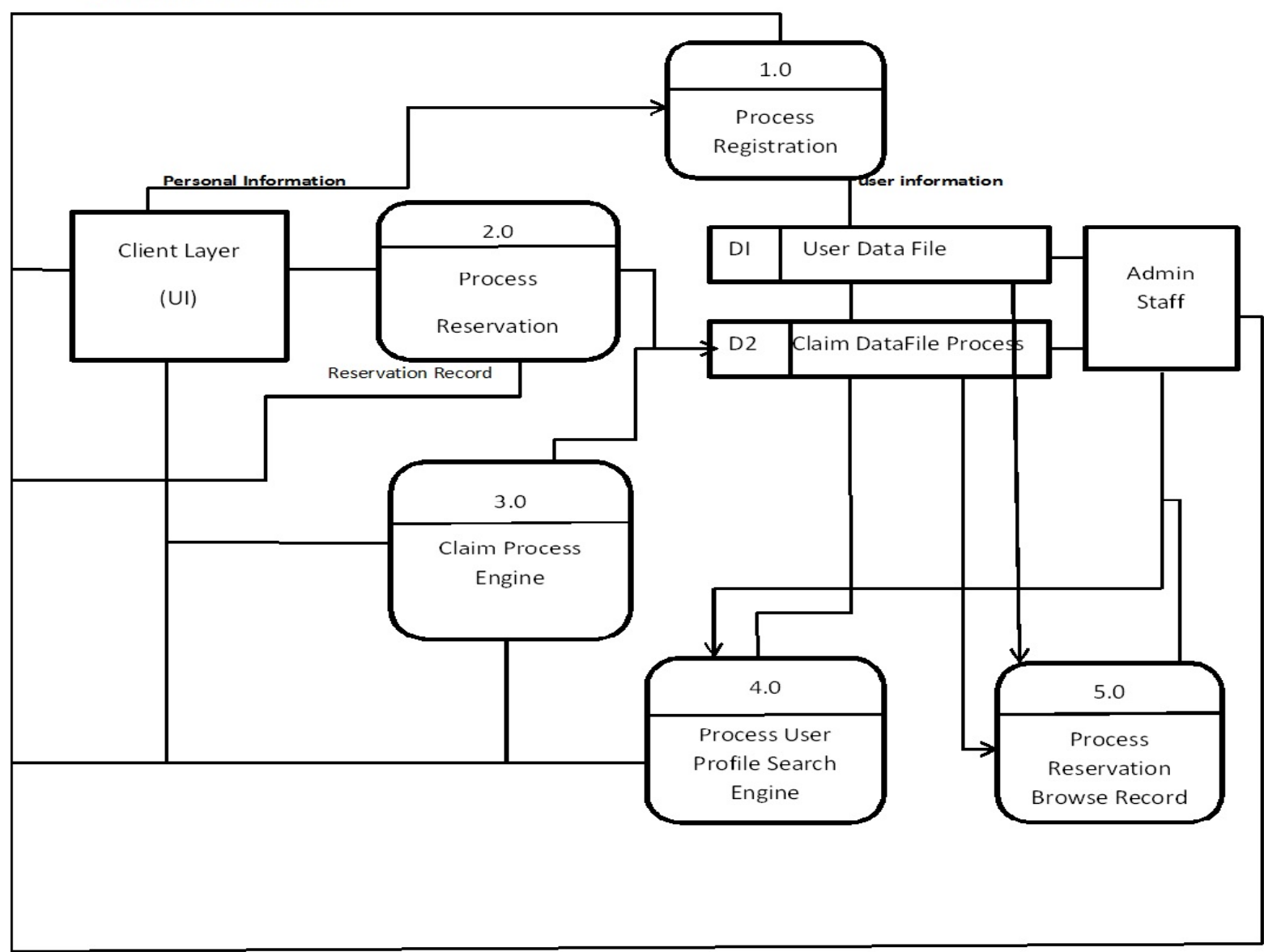

Fig.1 Dataflow Diagram Claim Process

\section{CONCLUSION}

This paper surveys diverse aspects of Insurancebased healthcare technologies and presents various health care network architectures and platforms that support access to the Insurance backbone and facilitate medical data.

\section{REFERENCES}

[1]. Bhat, Jain. Factor Affecting the Demand for Health Insurance in a Micro Insurance Scheme. 2006; Indian institute of management; Ahmedabad: W.P. No. 2006-0702.
[2]. Kundu S. Health Insurance - An Alternative Healthcare Financing Mechanism in Rural India. IJHD. 2009; 3(2).

[3]. Telecom Italia Lab, Java Agent Development Framework,http://jade.tilab.com/.

[4]. World Health Organization. Study of RSBY Health Insurance in India. AHPSR, Alliance for Health Policy and Systems Research; 2011; 2011/126289-0,

[5]. Abdullah L, Mohd R. IJCSI International Journal of Computer Science Issues. 2012; 9(1): No 2, 1694-0814 ISSN (Online).

[6]. Barick BL. Occupational health programs: the ounce of prevention paying off. Occup Health Saf. 1985; 38-42.

[7]. Khajehpour, Salari \& E. Raheleh, (2013) "Advancements and Trends in Medical Case-Based" 